\title{
Low-lying structure and shape evolution in neutron-rich Se isotopes
}

S. Chen,,${ }^{1,2,}$ P. Doornenbal, ${ }^{2}$ A. Obertelli, ${ }^{2,3}$ T. R. Rodríguez, ${ }^{4}$ G. Authelet, ${ }^{3}$ H. Baba, ${ }^{2}$ D. Calvet, ${ }^{3}$ F. Château, ${ }^{3}$ A. Corsi, ${ }^{3}$ A. Delbart, ${ }^{3}$ J.-M. Gheller, ${ }^{3}$ A. Giganon, ${ }^{3}$ A. Gillibert, ${ }^{3}$ V. Lapoux, ${ }^{3}$ T. Motobayashi, ${ }^{2}$ M. Niikura, ${ }^{5}$ N. Paul, ${ }^{3}$ J.-Y. Roussé,${ }^{3}$ H. Sakurai, ${ }^{2,5}$ C. Santamaria, ${ }^{3}$ D. Steppenbeck, ${ }^{2}$ R. Taniuchi, ${ }^{2,5}$ T. Uesaka, ${ }^{2}$ T. Ando, ${ }^{2,5}$ T. Arici, ${ }^{6}$ A. Blazhev, ${ }^{7}$ F. Browne, ${ }^{8}$ A. M. Bruce, ${ }^{8}$ R. Caroll, ${ }^{9}$ L. X. Chung, ${ }^{10}$ M. L. Cortés,${ }^{6,11}$ M. Dewald, ${ }^{7}$ B. Ding, ${ }^{12}$ F. Flavigny, ${ }^{13}$ S. Franchoo, ${ }^{13}$ M. Górska, ${ }^{6}$ A. Gottardo, ${ }^{13}$ A. Jungclaus,${ }^{14}$ J. Lee, ${ }^{15}$ M. Lettmann, ${ }^{11}$ B. D. Linh, ${ }^{10}$ J. Liu, ${ }^{15}$ Z. Liu, ${ }^{12}$ C. Lizarazo, ${ }^{6,11}$ S. Momiyama, ${ }^{2,5}$ K. Moschner, ${ }^{7}$ S. Nagamine, ${ }^{2,5}$ N. Nakatsuka, ${ }^{2,16}$ C. R. Nita, ${ }^{17}$ C. Nobs,${ }^{8}$ L. Olivier, ${ }^{13}$ R. Orlandi, ${ }^{18}$ Z. Patel, ${ }^{9}$ Zs. Podolyak, ${ }^{9}$ M. Rudigier, ${ }^{9}$ T. Saito, ${ }^{2,5}$ C. Shand, ${ }^{9}$ P.-A. Söderström, ${ }^{2}$ I. Stefan, ${ }^{13}$ V. Vaquero, ${ }^{14}$ V. Werner, ${ }^{11}$ K. Wimmer, ${ }^{5}$ and Z. Xu ${ }^{15}$

${ }^{1}$ School of Physics and State Key Laboratory of Nuclear Physics and Technology, Peking University, Beijing 100871, China

${ }^{2}$ RIKEN Nishina Center, Wako, Saitama 351-0198, Japan

${ }^{3}$ IRFU, CEA, Université Paris-Saclay, 91191 Gif-sur-Yvette, France

${ }^{4}$ Departamento de Física Teórica, Universidad Autónoma de Madrid, 28049, Madrid, Spain

${ }^{5}$ Department of Physics, University of Tokyo, 7-3-1 Hongo, Bunkyo, Tokyo 113-0033, Japan

${ }^{6}$ GSI Helmholtzzentrum für Schwerionenforschung GmbH, 64291 Darmstadt, Germany

${ }^{7}$ Institut für Kernphysik, Universität zu Köln, 50923 Köln, Germany

${ }^{8}$ School of Computing, Engineering and Mathematics, University of Brighton, Brighton BN2 4GJ, United Kingdom

${ }^{9}$ Department of Physics, University of Surrey, Guildford GU2 7XH, United Kingdom

${ }^{10}$ Institute for Nuclear Science and Technique, VINATOM, 179 Hoang Quoc Viet Road, Cau Giay, Hanoi, Vietnam

${ }^{11}$ Institut für Kernphysik, Technische Universität Darmstadt, 64289 Darmstadt, Germany

${ }^{12}$ Institute of Modern Physics, Chinese Academy of Sciences, Lanzhou 730000, China

${ }^{13}$ Institut de Physique Nucléaire, CNRS-IN2P3, Université Paris-Sud, Université Paris-Saclay, 91406 Orsay Cedex, France

${ }^{14}$ Instituto de Estructura de la Materia, CSIC, 28006 Madrid, Spain

${ }^{15}$ Department of Physics, The University of Hong Kong, Pokfulam, Hong Kong

${ }^{16}$ Department of Physics, Faculty of Science, Kyoto University, Kyoto 606-8502, Japan

${ }^{17}$ Horia Hulubei National Institute of Physics and Nuclear Engineering (IFIN-HH), R-077125 Bucharest, Romania

${ }^{18}$ Advanced Science Research Center, Japan Atomic Energy Agency, Tokai, Ibaraki, 319-1195, Japan

(Received 6 July 2016; revised manuscript received 29 March 2017; published 27 April 2017)

\begin{abstract}
Neutron-rich ${ }^{88,90,92,94} \mathrm{Se}$ isotopes were studied via in-beam $\gamma$-ray spectroscopy after nucleon removal reactions at intermediate energies at the Radioactive Isotope Beam Factory. Based on $\gamma-\gamma$ coincidence analysis, low-lying excitation level schemes are proposed for these nuclei, including the $2_{1}^{+}, 4_{1}^{+}$states and $2_{2}^{+}$states at remarkably low energies. The low-lying $2_{2}^{+}$states, along with other features, indicate triaxiality in these nuclei. The experimental results are in good overall agreement with self-consistent beyond-mean-field calculations based on the Gogny D1S interaction, which suggests both triaxial degree of freedom and shape coexistence playing important roles in the description of intrinsic deformations in neutron-rich Se isotopes.
\end{abstract}

DOI: 10.1103/PhysRevC.95.041302

Atomic nuclei are complex systems composed of interacting protons and neutrons. Nevertheless, their low-lying energy levels can often be simply described by rotations and vibrations around intrinsic shapes. Due to the underlying shell structure, governed by the effective nuclear interaction, such intrinsic shapes evolve with the number of nucleons in the system. Equilibrium shapes are frequently associated with the occurrence of shell gaps in Nilsson-like singleparticle levels around the Fermi level. When moving from closed-shell to open-shell nuclei, transitions from spherical to deformed shapes can be observed along isotopic and isotonic chains. Generally, ground-state deformations evolve smoothly as a function of nucleon number. In some regions of the nuclear chart, however, rapid shape transitions occur, indicating coexisting different intrinsic shapes competing at low excitation energy $[1,2]$.

\footnotetext{
*sidong.chen@ @iken.jp
}

The neutron-rich nuclei around $N \approx 60$ have been identified as a region of shape coexistence and shape transitions [2-6]. The $\mathrm{Zr}$ and $\mathrm{Sr}$ isotopes in this region exhibit sudden changes from almost spherical to highly deformed ground states at $N=60$, which belong to the most drastic shape transitions in the nuclear chart and were interpreted as intruders of coexisting deformed shapes [2]. Recent experimental studies of these isotopes have confirmed the competition of low-lying spherical and deformed configurations in ${ }^{96,98} \mathrm{Sr}$ [7] and established shape coexistence in ${ }^{94,96} \mathrm{Zr}$ [8,9]. Conversely, for the neutron-rich $\mathrm{Kr}$ isotopes, although evidence for a gradual increase of collectivity has been found up to $N=60$ [10-12], yielding no sudden change from spherical to strong deformation shapes, a possible prolate-oblate shape coexistence has been proposed for ${ }^{96} \mathrm{Kr}$ and heavier isotopes [5,12].

Experimental data and theoretical studies for the neutronrich Se isotopes are scarce. The heaviest isotope for which mass and $\beta$-decay half-life are established is ${ }^{90} \mathrm{Se}[13,14]$, while low-lying excited states have been observed only up 
to $N=53,54$ [15-17], both far away from the rapid onset of ground-state deformation found at $N \approx 60$ in $\mathrm{Sr}$ and $\mathrm{Zr}$ nuclei. The reported $2_{1}^{+}$energy of $886 \mathrm{keV}$ for ${ }^{88} \mathrm{Se}$ suggests a subshell effect at $N=56$ for ${ }^{90} \mathrm{Se}$ [16]. However, a search for subshell signatures from half-life measurements provided no support for the existence of an $N=56$ subshell [14]. In addition, a recent experimental study of ${ }^{86} \mathrm{Se}$ by $\gamma$-ray spectroscopy reported a possible $3^{+}$level, indicating an onset of $\gamma$ collectivity in ${ }^{86} \mathrm{Se}[18]$. Meanwhile, the $B(E 2)$ measurement and comparison with shell-model calculations suggest a triaxial shape in ${ }^{86} \mathrm{Se}$ [19]. Moving to more neutronrich Se isotopes, it is expected that nonaxial degrees of freedom play an important role in the description of deformation shapes [20]. Furthermore, systematic theoretical calculations in this region $[4,21]$ predict close-lying prolate and oblate minima in potential energy curves, originating from the downsloping $v h_{11 / 2}$ orbitals, which also suggest shape coexistence and ground-state shape transition in the Se isotopic chain.

The goal of this paper is twofold: (i) the identification of low-lying excited states of the very neutron-rich even isotopes ${ }^{88-94} \mathrm{Se}$ in order to assess their structures up to $N=60$; and (ii) the comparison of the experimental data with symmetry-conserving configuration mixing (SCCM) calculations that indicate that this region of the isotopic chain shows evidences of sudden prolate-oblate shape transitions and shape coexistence.

The experiment was carried out at the Radioactive Isotope Beam Factory, operated by the RIKEN Nishina Center and the Center for Nuclear Study of the University of Tokyo. A ${ }^{238} \mathrm{U}$ primary beam, accelerated to an energy of $345 \mathrm{MeV} / \mathrm{u}$, was provided with an average intensity of $30 \mathrm{pnA}$. Secondary radioactive isotope beams were produced by in-flight fission of the primary beam in a 3-mm-thick Be production target placed at the entrance of the fragment separator BigRIPS [22]. The isotopes of interest were selected and separated with the $B \rho-\Delta E-B \rho$ method and identified on an event-by-event basis by a TOF- $B \rho-\Delta E$ measurement [23]. Two BigRIPS settings were applied and centered on ${ }^{89} \mathrm{As}$ and ${ }^{95} \mathrm{Br}$, respectively. The average intensities of the ${ }^{89} \mathrm{Se}$ and ${ }^{91,94,95} \mathrm{Br}$ isotopes, which were used to produce the Se isotopes of interest, were measured to be $618,1280,182$, and 50 particles per second, respectively, and impinged on a 99(1)-mm-thick [725(7)-mg/cm $\left.{ }^{2}\right]$ liquid hydrogen reaction target. Their energies in front of the reaction target were $\sim 270 \mathrm{MeV} / \mathrm{u}$ and decreased to $\sim 180 \mathrm{MeV} / \mathrm{u}$ at the exit of the reaction target. Reaction residues were collected and unambiguously identified by the ZeroDegree spectrometer [22], employing a similar identification method as described for BigRIPS.

Surrounding the liquid hydrogen target was a 300-mm-long cylindrical time projection chamber (TPC) of the MINOS ensemble [24]. The usage of the thick liquid hydrogen target required the employment of the TPC to track the protons for $(p, 2 p)$ and other reactions and to reconstruct their vertex position for the Doppler correction. An efficiency of $>91 \%$ for good vertex reconstruction of the ${ }^{91} \operatorname{Br}(p, 2 p){ }^{90} \mathrm{Se}$ channel was obtained with a spatial resolution of $\sim 5 \mathrm{~mm}$ FWHM. Examples of reconstructed vertices from two proton trajectories for $(p, 2 p)$ knock-out reactions are illustrated in
Refs. [24,25]. In the case of $(p, p n)$ channels, the beam and scattered proton trajectories were used to determine the reaction vertex. Deexcitation $\gamma$ rays were measured by the DALI2 detector array [26,27], which consisted of 186 $\mathrm{NaI}(\mathrm{Tl})$ scintillators. A full-energy peak detection efficiency of $35 \%$ (23\%), including add-back, was simulated for 500-keV $(1-\mathrm{MeV}) \gamma$ rays emitted from the target center at a beam energy of $250 \mathrm{MeV} / \mathrm{u}$ with the GEANT4 framework [28]. Energy calibrations were performed for each BigRIPS-ZeroDegree setting using ${ }^{60} \mathrm{Co},{ }^{137} \mathrm{Cs},{ }^{88} \mathrm{Y}$, and ${ }^{133} \mathrm{Ba}$ sources, resulting in a calibration error of $2 \mathrm{keV}$ in the range of 350-1300 keV and an energy resolution of 9\% (6\%) FWHM at $662 \mathrm{keV}$ (1.33 MeV), consistent with Refs. [26,27].

The Doppler-corrected spectra for ${ }^{88-94} \mathrm{Se}$ are shown in Fig. 1, assuming all $\gamma$ rays were emitted from the reaction vertex reconstructed by MINOS. However, the lifetimes of the excited states influenced the Doppler correction. This effect was considered when obtaining the DALI2 response functions from GEANT4 simulations by assuming lifetimes based on the theoretical approach discussed later and included in the errors for the energy determinations. Furthermore, the validity of the Doppler correction was verified using the well-known $4_{1}^{+} \rightarrow 2_{1}^{+}$and $2_{1}^{+} \rightarrow 0_{\text {g.s. }}^{+}$transitions of ${ }^{94} \mathrm{Kr}[11,29]$ following $(p, p n)$ reactions, which yielded less than $1 \mathrm{keV}$ deviation to the literature values. Each spectrum was fitted with DALI2 response functions added on top of a double-exponential background. $\gamma-\gamma$ coincidences were used to establish level schemes for decays observed in the single spectra. Examples of this analysis as well as the established level schemes are given in the insets of Fig. 1. However, the current measurement was not sensitive to spin parities. Thus, their assignments were based on the systematics of the isotopic chain and the comparison with other studies of knock-out reactions [25,31,32].

For ${ }^{88} \mathrm{Se}$, the previously reported $\gamma$-ray line at $886 \mathrm{keV}$ assigned to the $2_{1}^{+} \rightarrow 0_{\text {g.s. }}^{+}$transition [16] was not observed. Instead, the most intense $\gamma$-ray transition after efficiency correction was found at $580(8) \mathrm{keV}$, which was assigned to the $2_{1}^{+} \rightarrow 0_{\text {g.s. }}^{+}$decay as in other nuclei studies [25,31,32]. Toward the high-energy side, a tail was observed and fitted by another transition at 646(15) keV. Two other transitions were observed at $971(10)$ and $1232(13) \mathrm{keV}$. The $\gamma-\gamma$ analysis showed that the 646- and 971-keV transitions were in coincidence with the $2_{1}^{+}$state, while, within the uncertainties, the $1232-\mathrm{keV}$ transition was in good agreement with the sum of the 580- and 646-keV transitions. Therefore, the latter was assigned to the $2_{2}^{+} \rightarrow 0_{\text {g.s. }}^{+}$transition, while the former were assigned to the $2_{2}^{+} \rightarrow 2_{1}^{+}$and $4_{1}^{+} \rightarrow 2_{1}^{+}$transitions.

Five transitions were observed at 419(8), 548(9), 691(7), $960(15)$, and $1075(24) \mathrm{keV}$ for ${ }^{90} \mathrm{Se}$. As in the other cases, the most intense transition, here at $548 \mathrm{keV}$, was assigned to the $2_{1}^{+} \rightarrow 0_{\text {g.s. }}^{+}$decay. The $\gamma-\gamma$ analysis revealed only the $960-\mathrm{keV}$ peak was not in coincidence with the $548-\mathrm{keV}$ transition [see Fig 1(b) inset], while it matched the sum of the 419- and 548-keV decays within errors. Thus, we assigned the 960and $419-\mathrm{keV}$ transitions to the $2_{2}^{+} \rightarrow 0_{\text {g.s. }}^{+}$and $2_{2}^{+} \rightarrow 2_{1}^{+}$ transitions, respectively. The $691-$ and $1075-\mathrm{keV}$ transitions were placed on top of the $2_{1}^{+}$state in the proposed level 


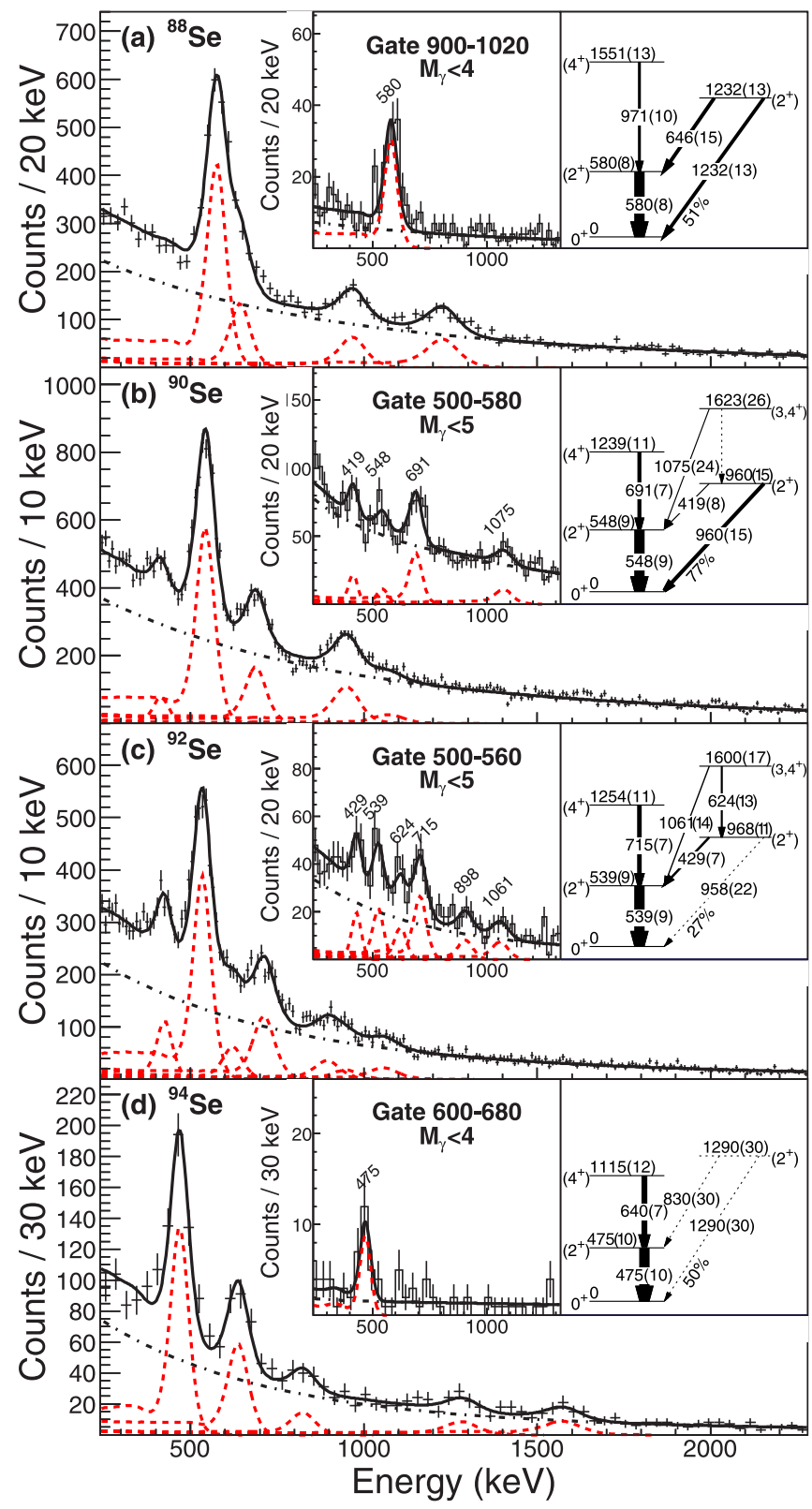

FIG. 1. Doppler-corrected $\gamma$-ray spectra. Each spectrum is from a single reaction channel: ${ }^{89} \mathrm{Se}(p, p n){ }^{88} \mathrm{Se}(\mathrm{a}),{ }^{91} \mathrm{Br}(p, 2 p){ }^{90} \mathrm{Se}(\mathrm{b})$, ${ }^{94} \mathrm{Br}(p, 2 p n){ }^{92} \mathrm{Se}(\mathrm{c}),{ }^{95} \mathrm{Br}(p, 2 p){ }^{94} \mathrm{Se}(\mathrm{d})$. The spectra were fitted with simulated response functions (red) added on top of doubleexponential background (black). In the insets, examples of the $\gamma-\gamma$ coincidence analysis and deduced level schemes are presented, the widths of the arrows reflect relative intensities of transitions, dashed lines are used for very weak transitions. The self-coincidences in (b) and (c) insets originate from Compton events of high-energy transitions.

scheme, with the $1239-\mathrm{keV}$ state assigned to the $4_{1}^{+}$and the $1623-\mathrm{keV}$ state tentatively assigned to a $\left(3,4^{+}\right)$state, based on the comparison to ${ }^{92} \mathrm{Se}$ discussed below. A $\left(3,4^{+}\right) \rightarrow 2_{2}^{+}$ transition remained unobserved, possibly because it coincided energetically with the $4_{1}^{+} \rightarrow 2_{1}^{+}$transition.
In a previous isomer study, $\gamma$-ray lines for ${ }^{92} \mathrm{Se}$ were reported at 503, 539, and $898 \mathrm{keV}$, but no level scheme was established [33]. In the present work, seven transitions were found at 429(7), 539(9), 624(13), 715(7), 898(15), 958(22), and $1061(14) \mathrm{keV}$, with the 539-keV transition assigned to the $2_{1}^{+} \rightarrow 0_{\text {g.s. }}^{+}$and the $715-\mathrm{keV}$ transition assigned to the $4_{1}^{+} \rightarrow$ $2_{1}^{+}$, based on the relative intensities. It is noted that owing to the accurately known transition at $898 \mathrm{keV}$, the peak at 958(22) $\mathrm{keV}$ could be identified, which was revealed as a doublet with the 898-keV transition in the form of a high-energy tail. Note that lifetime effects may only lead to tails toward the lower energies and calculations using the theoretical $B(E 2)$ values suggest all excited states should have lifetimes shorter than $50 \mathrm{ps.}$ Based on the $\gamma-\gamma$ analysis and the sum of transition energies, we assigned the 429- and 958-keV $\gamma$-ray decay from the $2_{2}^{+}$ state at $968(11) \mathrm{keV}$, and the $624-$ and $1061-\mathrm{keV} \gamma$-ray decay from a level at $1600(17) \mathrm{keV}$, which was tentatively assigned to be the $\left(3,4^{+}\right)$from comparison to the level scheme of ${ }^{86} \mathrm{Se}$ [18]. The 898-keV transition could not be placed in the proposed level scheme. It is further noted that the coincidence spectrum features a reduced self-coincidence with the $2_{1}^{+}$decay due to Compton events originating from high-energy transitions.

For the most exotic isotope studied here, ${ }^{94} \mathrm{Se}$, two clear transitions were seen at 475(10) and 640(7) $\mathrm{keV}$ and in coincidence with each other. They were assigned to the $2_{1}^{+} \rightarrow$ $0_{\text {o.s. }}^{+}$and $4_{1}^{+} \rightarrow 2_{1}^{+}$transitions based on relative intensities. In addition, three peak-like structures were observed at 830(30), 1290(30), and 1580(30) keV. Standard significance tests [34] yielded $3.6 \sigma, 3.0 \sigma$, and $3.7 \sigma$, respectively, for these candidate peaks (we required a significance of at least $5 \sigma$ for an assignment of a new transition). The $1290 \mathrm{keV}$ matches well the sum of 475 and $830 \mathrm{keV}$ within errors, thus a candidate $2_{2}^{+}$ level at 1290(30) $\mathrm{keV}$ was placed in the level scheme. Note that a $\gamma-\gamma$ analysis could not be performed for these candidate peaks due to limited statistics.

The systematics of $E\left(2_{1}^{+}\right), R_{4 / 2}$ ratio, and $E\left(2_{2}^{+}\right)$for Se in comparison with $\mathrm{Zr}$, $\mathrm{Sr}$, and $\mathrm{Kr}$ isotopes are shown in Fig. 2 from $N=50$ to 60 . A gradual decrease of $E\left(2_{1}^{+}\right)$for the Se isotopes is apparent, yielding no indication for a subshell closure at $N=56$ nor a sharp rise of deformation at $N=60$. The $R_{4 / 2}$ ratio increases from $N=50$ to 54 , followed by a drop at $N=56$, then stays roughly constant at values around 2.3 until $N=60$. When comparing with other isotopic chains, both the $E\left(2_{1}^{+}\right)$and $R_{4 / 2}$ pattern are at variance with the $\mathrm{Zr}$ and $\mathrm{Sr}$ isotopes and strongly resemble the trend of the $\mathrm{Kr}$ isotopes [11,12,29]. More interestingly, the assigned $2_{2}^{+}$ levels keep decreasing until $N=56,58$, then show a possible increase at $N=60$, especially for $N=56,58$, the $E\left(2_{2}^{+}\right)$are among the lowest in this mass region [see Fig 2(c)] and thus indicate $\gamma$-soft or triaxial features in these nuclei. Further, the $B\left(E 2 ; 2_{2}^{+} \rightarrow 2_{1}^{+}\right) / B\left(E 2 ; 2_{2}^{+} \rightarrow 0_{1}^{+}\right)$ratios calculated from the experimental branching ratios and level energies, assuming negligible $B(M 1)$ contributions, give values of 24(4), 20(4), 161(57), and 9(5) for ${ }^{88,90,92,94} \mathrm{Se}$, respectively. These large ratios are consistent with the $\mathrm{O}(6)$ limit in the interacting boson approximation (IBA) model [35].

In the following, the experimental results are compared with a symmetry-conserving configuration mixing (SCCM) 

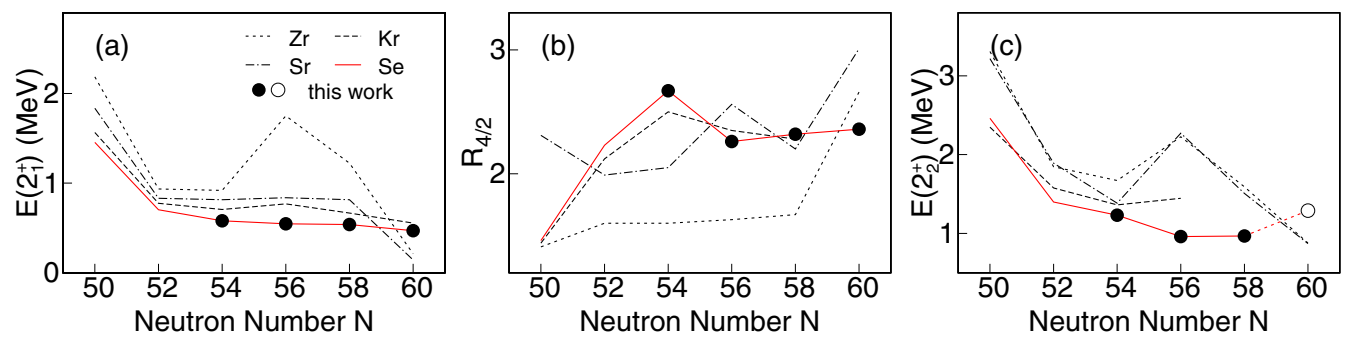

FIG. 2. Systematics of $E\left(2_{1}^{+}\right)$in $\mathrm{Zr}, \mathrm{Sr}, \mathrm{Kr}$, and Se isotopes (a), the same for their $R_{4 / 2}=E\left(4_{1}^{+}\right) / E\left(2_{1}^{+}\right)$ratio (b), and their $E\left(2_{2}^{+}\right)$(c). Open symbols are for candidate states. Data are from this work and Refs. [11,16,29,30].

calculation with Gogny D1S effective interaction [36,37]. In this framework, each individual nuclear state is defined as the linear combination of multiple intrinsic many-body states with different quadrupole (axial and triaxial) shapes. Further details are described in Refs. [5,38-40]. In addition, only time-reversal (static) and parity symmetric intrinsic shapes were considered, i.e., cranked or octupole deformed states were not included. Therefore, a systematic stretching of the levels with respect to the experimental values is expected [41] and negative parity bands cannot be described. We note that in the $(p, 2 p)$ reactions studied in this paper, direct population of negative parity states requires proton knockout from the $g_{9 / 2}$ orbital. This orbital is expected to be only weakly occupied in the ground states of the $\mathrm{Br}$ isotopes, as inferred from the recent calculations for the $\mathrm{Zr}$ isotopes [6]. Therefore, the present theory is considered sufficient to describe all observed levels.

In Fig. 3, the theoretical predicted level energies are compared with experimental results for ${ }^{86-94}$ Se. Low-lying $2_{2}^{+}$ states below the $4_{1}^{+}$levels are well reproduced for ${ }^{86-92} \mathrm{Se}$ and an increase of $E\left(2_{2}^{+}\right)$is also predicted for ${ }^{94} \mathrm{Se}$. As remarked above, a general stretching of the theoretical predictions is observed, mainly due to the privileged exploration of the ground-state energy in the present variational process without cranking states [41]. Despite the stretching, the present SCCM calculation describes rather well the trends of the experimental energies for the $2_{1}^{+}, 4_{1}^{+}$, and $2_{2}^{+}$states, although some differences are found, e.g., the decrease of the $E\left(2_{1}^{+}\right)$

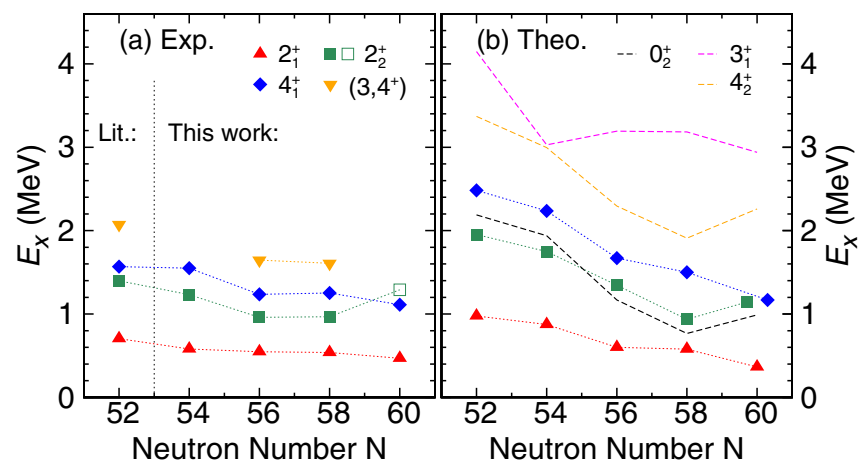

FIG. 3. Comparison of experimental and theoretical $E\left(2_{1}^{+}\right)$, $E\left(4_{1}^{+}\right), E\left(2_{2}^{+}\right)$, and $E\left(3,4^{+}\right)$from ${ }^{86} \mathrm{Se}$ to ${ }^{94} \mathrm{Se}$. Open symbols are for candidate states. Data are from this work and Ref. [30]. from ${ }^{88}$ Se to ${ }^{90} \mathrm{Se}$ and of the $E\left(2_{2}^{+}\right)$from ${ }^{90} \mathrm{Se}$ to ${ }^{92} \mathrm{Se}$ instead of the flat experimental trend. The present SCCM calculations also yield large $B\left(E 2 ; 2_{2}^{+} \rightarrow 2_{1}^{+}\right) / B\left(E 2 ; 2_{2}^{+} \rightarrow 0_{1}^{+}\right)$ratios of $49,11,41$, and 5 for ${ }^{88,90,92,94} \mathrm{Se}$, respectively. These calculated ratios are not as extensive as measured, yet the systematic trend is correctly obtained. Further, calculated $B\left(E 2 ; 2_{1}^{+} \rightarrow\right.$ $0_{\mathrm{gs}}^{+}$) values can be compared to available experimental data. The calculations yield values of $411 e^{2} \mathrm{fm}^{4}$ and $546 e^{2} \mathrm{fm}^{4}$ compared to experimental values of 210(30) $e^{2} \mathrm{fm}^{4}$ and $438_{-171}^{+259} e^{2} \mathrm{fm}^{4}$ for ${ }^{84,86} \mathrm{Se}$ [19], respectively. The discrepancy for the semi-magic ${ }^{84} \mathrm{Se}$ is expected due to the relevance of quasiparticle excitations not taken into account in the present SCCM framework. In addition, the present SCCM calculation favors a $4_{2}^{+}$assignment for the $\left(3,4^{+}\right)$levels in ${ }^{90,92} \mathrm{Se}$. Predicted $3_{1}^{+}$states, which belong to the $\gamma$ bands, lie about $1 \mathrm{MeV}$ higher in energy [see Fig. 3(b)].

To gain insight into the intrinsic deformation, we display in Fig. 4 the potential energy surfaces (PESs) for ${ }^{86-94} \mathrm{Se}$ together with the collective wave functions (c.w.f.) for the $0_{\text {g.s. }}^{+}, 2_{1}^{+}$, and $2_{2}^{+}$states. All c.w.f. have maxima in probabilities at $\beta_{2} \sim 0.2-0.3$ but quite different behaviors in the $\gamma$ degree of freedom. In ${ }^{86,88} \mathrm{Se}$, both PES and c.w.f. are predicted to be extending in the $\gamma$ direction, in good agreement with the $\mathrm{O}(6)$ limit. However, going to ${ }^{90,92,94} \mathrm{Se}$, two distinct minima with similar absolute energies are predicted in the PESs, hinting a possible shape coexistence in these nuclei. By looking into the c.w.f., the yrast states $\left(4_{1}^{+}\right.$not shown) in ${ }^{90} \mathrm{Se}$ are predicted to be prolate deformed, while the low-lying $2_{2}^{+}$state features a competing oblate shape. Conversely, ${ }^{94} \mathrm{Se}$ exhibits an oblate yrast band and a prolate $2_{2}^{+}$state. For ${ }^{92} \mathrm{Se}$, the calculation predicts a $\gamma$-soft ground state which evolves to a stabilized oblate shape for the $4_{1}^{+}$state, while the $2_{2}^{+}$state exhibits a prolate- $\gamma$-soft deformation mixing with an oblate configuration. Overall, when following the Se isotopic chain, the SCCM calculations show an intriguing shape transition: the yrast states evolve from prolate $\left({ }^{90} \mathrm{Se}\right)$ to oblate $\left({ }^{94} \mathrm{Se}\right)$ through a transitional $\gamma$-soft $\left({ }^{92} \mathrm{Se}\right)$ shape, and the $2_{2}^{+}$states, conversely to the yrast states, undergo an oblate- $(\gamma$-soft $)$-prolate transition. Quantitatively, from ${ }^{90} \mathrm{Se}$ to ${ }^{94} \mathrm{Se}$, the theoretical calculated $Q_{\mathrm{sp}}$ evolves from -42 to $+55 e \mathrm{fm}^{2}$ for the $2_{1}^{+}$state and from +43 to $-46 e \mathrm{fm}^{2}$ for the $2_{2}^{+}$state. These transitions can be understood in the view of the $\mathrm{O}(6)$ limit in the IBA model: As pointed out in Refs. [42,43], the $\mathrm{O}(6)$ limit is the critical point of a first-order prolate-oblate shape-phase transition. The Se isotopes studied here were all 


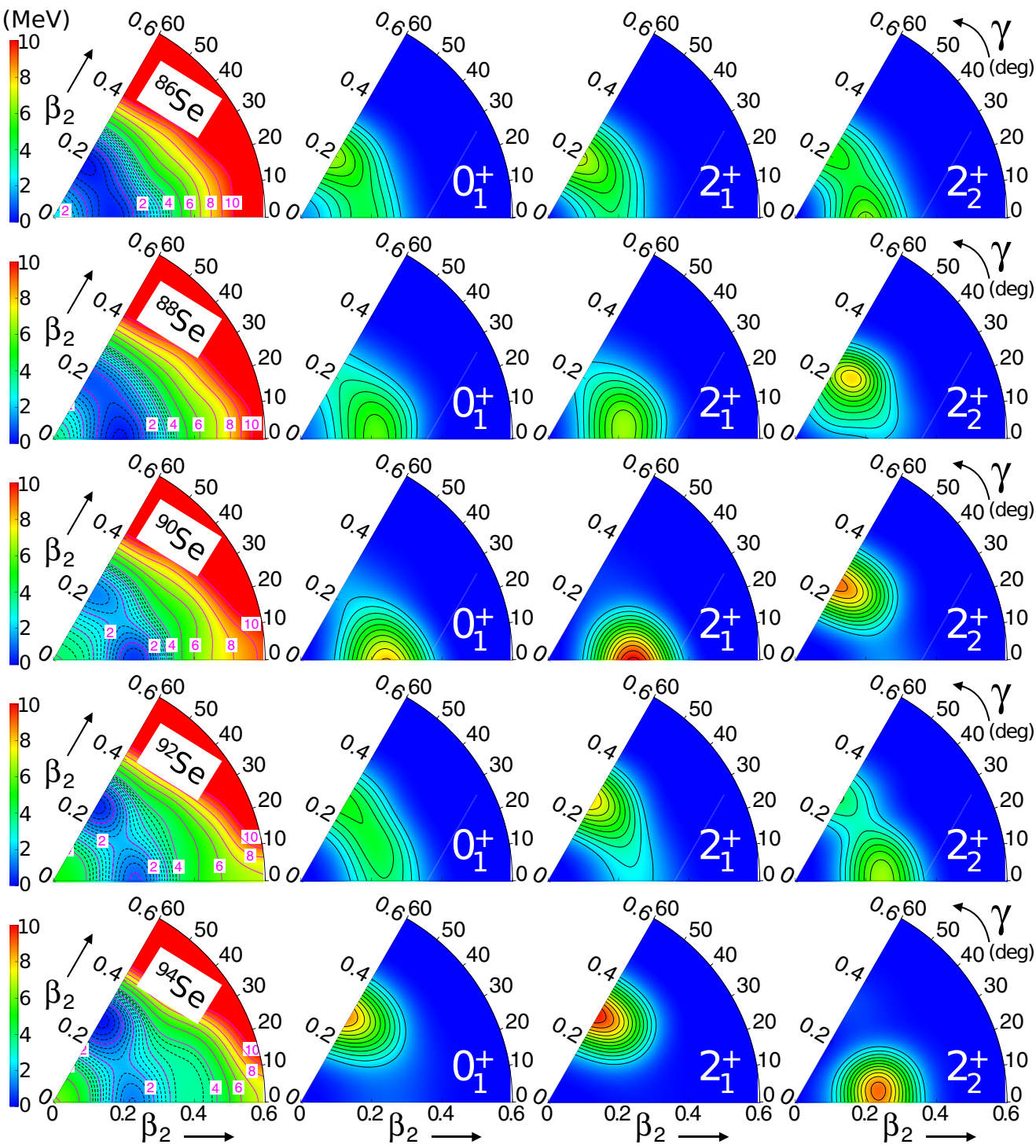

FIG. 4. Potential energy surfaces and collective wave functions (normalized to unity) for the $0_{\text {g.s. }}^{+}, 2_{1}^{+}, 2_{2}^{+}$states of even ${ }^{86-94}$ Se. The color code $($ red $=0.15$, blue $=0.00$ ) in c.w.f. represents the weight of different deformations in the composition of a state. The contour lines are separated by 0.01 .

indicative of being located around the $\mathrm{O}(6)$ limit; however, it is difficult to be exactly at the critical point where the $\gamma$ degree of freedom has no global minimum. Finally, the calculations predicted intruder $0_{2}^{+}$states strongly connected to the $2_{2}^{+}$and $4_{2}^{+}$states [see Fig. 3(b)], which have not been experimentally observed. The present measurement could be insensitive to those states due to their proximity to the $2_{2}^{+}$states, resulting in weak $2_{2}^{+} \rightarrow 0_{2}^{+}$transition rates and branching ratios.

In summary, low-lying excited states in ${ }^{88} \mathrm{Se}$ and for the first time in ${ }^{90,92,94} \mathrm{Se}$ were studied. In total, 18 transitions from 13 newly proposed excitation levels were reported. A smooth, shallow drop of $E\left(2_{1}^{+}\right)$was observed up to $N=60$, yielding no evidence for a subshell closure at $N=56$ nor a rapid onset of deformation at $N=60$. It was found that all the proposed $2_{2}^{+}$levels lie below or close to the $4_{1}^{+}$states and their energies decrease continuously to below $1 \mathrm{MeV}$ for ${ }^{90,92} \mathrm{Se}$, indicating triaxiality in these nuclei. The obtained results are in reasonable agreement with beyond-mean-field calculations based on the SCCM approach, which point out the important roles of the triaxial degree of freedom and shape coexistence for a proper description of the neutron-rich $\mathrm{Se}$ isotopes. Strikingly low-lying $0_{2}^{+}$states were predicted in the same theoretical model. Finding these states is a key step forward in future experiments, while "safe" energy Coulomb excitation, though presumably beyond reach at presently existing facilities, may confirm the predicted deformed shapes in the Se isotopes.

We express our gratitude to the RIKEN Nishina Center accelerator staff for providing the stable and high-intensity uranium beam and to the BigRIPS team for operating the secondary beams. The development of MINOS and the core MINOS team were supported by the European Research Council through the ERC Grant No. MINOS-258567. This 
work was supported by the IPA program at RIKEN Nishina Center, by GSI-Darmstadt computing facilities and Ministerio de Economía y Competitividad under Contract FIS-201453434 and Programa Ramón y Cajal 2012 No. 11420, by the German BMBF Grants No. 05P15PKFNA, No. 05P12RDFN8, and No. 05P15RDFN1, by Ministerio de Economy Com- petitividad under Contract FPA2014-57196-C5-4-P, by the Hundred-Talent Program at Chinese Academy of Sciences, by the National Natural Science Foundation of China under Grant No. 11405224, by the Japan Society for the Promotion of Science under Grant No. 2604327, and by the Vietnam Ministry of Science and Technology.
[1] K. Heyde, J. Jolie, R. Fossion, S. De Baerdemacker, and V. Hellemans, Phys. Rev. C 69, 054304 (2004).

[2] K. Heyde and J. L. Wood, Rev. Mod. Phys. 83, 1467 (2011).

[3] K. Sieja, F. Nowacki, K. Langanke, and G. Martínez-Pinedo, Phys. Rev. C 79, 064310 (2009).

[4] P. Sarriguren, Phys. Rev. C 91, 044304 (2015).

[5] T. R. Rodríguez, Phys. Rev. C 90, 034306 (2014).

[6] T. Togashi, Y. Tsunoda, T. Otsuka, and N. Shimizu, Phys. Rev. Lett. 117, 172502 (2016).

[7] E. Clément et al., Phys. Rev. Lett. 116, 022701 (2016).

[8] A. Chakraborty et al., Phys. Rev. Lett. 110, 022504 (2013).

[9] C. Kremer et al., Phys. Rev. Lett. 117, 172503 (2016).

[10] S. Naimi et al., Phys. Rev. Lett. 105, 032502 (2010).

[11] M. Albers et al., Phys. Rev. Lett. 108, 062701 (2012).

[12] M. Albers et al., Nucl. Phys. A 899, 1 (2013).

[13] M. Wang, G. Audi, A. H. Wapstra, F. G. Kondev, M. MacCormick, X. Xu, and B. Pfeiffer, Chin. Phys. C 36, 1603 (2012).

[14] M. Quinn et al., Phys. Rev. C 85, 035807 (2012).

[15] T. Rzaca-Urban, M. Czerwiński, W. Urban, A. G. Smith, I. Ahmad, F. Nowacki, and K. Sieja, Phys. Rev. C 88, 034302 (2013).

[16] E. F. Jones et al., Phys. Rev. C 73, 017301 (2006).

[17] A. Korgul et al., Phys. Rev. C 92, 054318 (2015).

[18] T. Materna et al., Phys. Rev. C 92, 034305 (2015).

[19] J. Litzinger et al., Phys. Rev. C 92, 064322 (2015).

[20] K. Sieja, T. R. Rodríguez, K. Kolos, and D. Verney, Phys. Rev. C 88, 034327 (2013).

[21] J. P. Delaroche, M. Girod, J. Libert, H. Goutte, S. Hilaire, S. Péru, N. Pillet, and G. F. Bertsch, Phys. Rev. C 81, 014303 (2010).

[22] T. Kubo et al., Prog. Theor. Exp. Phys. 2012, 03 C003 (2012).

[23] N. Fukuda, T. Kubo, T. Ohnishi, N. Inabe, H. Takeda, D. Kameda, and H. Suzuki, Nucl. Instrum. Methods Phys. Res., Sect. B 317, 323 (2013).
[24] A. Obertelli et al., Eur. Phys. J. A 50, 8 (2014).

[25] C. Santamaria et al., Phys. Rev. Lett. 115, 192501 (2015).

[26] P. Doornenbal, Prog. Theor. Exp. Phys. 2012, 03 C004 (2012).

[27] S. Takeuchi, T. Motobayashi, Y. Togano, M. Matsushita, N. Aoi, K. Demichi, H. Hasegawa, and H. Murakami, Nucl. Instrum. Methods Phys. Res., Sect. A 763, 596 (2014).

[28] S. Agostinelli et al., Nucl. Instrum. Methods in Phys. Res. A 506, 250 (2003).

[29] T. Rzaca-Urban et al., Eur. Phys. J. A 9, 165 (2000).

[30] http://www.nndc.bnl.gov/ensdf/.

[31] P. Doornenbal et al., Phys. Rev. Lett. 111, 212502 (2013).

[32] N. Paul et al., Phys. Rev. Lett. 118, 032501 (2017).

[33] D. Kameda et al., Phys. Rev. C 86, 054319 (2012).

[34] K. Weise, K. Hübel, E. Rose, M. Schläger, D. Schrammel, M. Täschner, and R. Michel, Radiat. Prot. Dosimetry 121, 52 (2006).

[35] R. F. Casten, A. Aprahamian, and D. D. Warner, Phys. Rev. C 29, 356 (1984).

[36] J. Dechargé and D. Gogny, Phys. Rev. C 21, 1568 (1980).

[37] J. Berger, M. Girod, and D. Gogny, Nucl. Phys. A 428, 23 (1984)

[38] T. R. Rodríguez and J. L. Egido, Phys. Rev. C 81, 064323 (2010).

[39] M. Bender and P.-H. Heenen, Phys. Rev. C 78, 024309 (2008)

[40] J. M. Yao, J. Meng, P. Ring, and D. Vretenar, Phys. Rev. C 81, 044311 (2010).

[41] M. Borrajo, T. R. Rodríguez, and J. L. Egido, Phys. Lett. B 746, 341 (2015).

[42] J. Jolie, R. F. Casten, P. von Brentano, and V. Werner, Phys. Rev. Lett. 87, 162501 (2001).

[43] J. Jolie, P. Cejnar, R. F. Casten, S. Heinze, A. Linnemann, and V. Werner, Phys. Rev. Lett. 89, 182502 (2002). 